\title{
Large Fibroid arising from Mullerian Remnant Mimicking as Ovarian Neoplasm in a Woman with MRKH Syndrome
}

\author{
Soma Singh, Baidyanath Chakravarty, Manju Chakravarty, Astha Chakravarty
}

\section{ABSTRACT}

In this article, a large leiomyoma with degenerative changes arising from the rudimentary uterine knob in a patient with MayerRokitansky-Kuster-Hauser (MRKH) syndrome and mimicking an ovarian neoplasm is reported.

The patient was a 39-year-old woman, known case of MRKH with vaginal aplasia who presented with pain abdomen, loss of appetite and weight. On physical examination, a large pelvic mass was detected. A provisional diagnosis of left ovarian neoplasm was made on USG and CT scan for which an exploratory laparotomy was performed. Finally, it was diagnosed as a case of multiple leiomyomas with hyaline degeneration on histopathological examination.

Myoma arising from a rudimentary uterine knob/anlage is a rare finding but should be considered in the differential diagnosis of pelvic mass in patients with MRKH syndrome.

Keywords: Leiomyoma, Mayer-Rokitansky-Kuster-Hauser (MRKH) syndrome, Mullerian remnant.

How to cite this article: Singh S, Chakravarty B, Chakravarty M, Chakravarty A. Large Fibroid arising from Mullerian Remnant Mimicking as Ovarian Neoplasm in a Woman with MRKH Syndrome. Int J Infertility Fetal Med 2012;3(1):30-32.

Source of support: Nil

Conflict of interest: None declared

\section{INTRODUCTION}

Mayer-Rokitansky-Kuster-Hauser (MRKH) syndrome is a congenital abnormality of the genital tract and affects one in 4000 to 5000 liveborn females. ${ }^{1}$ The etiology of the syndrome remains unclear, and a multifactorial mode of inheritance has been proposed, including genetic and environmental factors. It usually presents with primary amenorrhea during adolescence. Concurrent association of pelvic mass with mullerian agenesis can be a diagnostic dilemma. Occurrence of myoma arising from a mullerian remnant is an extremely rare finding and only few cases have been reported so far in the literature. ${ }^{2-16}$ To the best of our knowledge, only one such case of mullerian duct remnant leiomyoma with hyaline degeneration mimicking ovarian neoplasm by USG scan has been reported in the past. ${ }^{7}$

Date of Received: 31-12-11

Date of Acceptance: 12-01-12

Date of Publication: January 2012

\section{CASE REPORT}

A 39-year-old unmarried, nulliparous female who was a known case of MRKH syndrome with vaginal agenesis with recent diagnoses of type II DM, presented with complaints of loss of weight and appetite since 3 months and pain in lower abdomen of 5 days duration. There were no associated urinary and bowel complaints. At 19 years of age, she was diagnosed with MRKH syndrome, and diagnostic laparoscopy had been performed. At laparoscopy, two mullerian knobs on either side of pelvis, two rudimentary fallopian tubes and normal ovaries were seen. Her karyotype was normal (XX). She had been advised for vaginoplasty which she refused and was lost to follow-up.

On physical examination, her BMI was 21.08, mild pallor was present and secondary sexual characters were adult female type. Thyroid was not enlarged. On abdominal examination, a lump was visible in the hypogastrium which on palpation, was nontender, firm to hard in consistency with slightly irregular surface and lower border of the lump was not reachable. It measured approximately $10 \times 8 \mathrm{~cm}$, with restricted sideways mobility. There was no free fluid in abdomen. Her external genitalia were normal female type. Vagina was absent and, on rectal examination, lower border of the pelvic mass could be felt. Laboratory investigations showed mild anemia (hemoglobin-10 gm\%), CA-125 was 14.5 U/ml (normal). USG abdomen revealed a huge solid hypoechoic, heterogeneous SOL $(11.31 \times 9.97 \mathrm{~cm})$ with irregular margin in pelvis, suspicious of ovarian neoplastic mass and mild hepatomegaly. CT scan of abdomen showed a multilobulated heterogeneous enhancing mass $(11.7 \times$ $10.6 \mathrm{~cm}$ ) in pelvis (left ovarian tumor). Uterus could not been seen and right ovary was normal (Fig. 1). It also showed mild hydroureter, hydronephrosis on left side and mild hepatomegaly. Our provisional diagnosis was of left-sided ovarian tumor and decision was taken for exploratory laparotomy. Abdomen was opened by low transverse incision and a huge tumor was seen occupying the entire pelvis, encroaching into left broad ligament and sacral hollow. Right-sided mullerian knob and ovary appeared normal but left-sided ovary could not be visualized. The tumor was solid in consistency, free of adhesions to neighboring structures and seemed to arise from left mullerian knob. There was no free fluid in the abdomen and no metastatic lesions seen. Both uterine knobs and tumor were removed uneventfully (Fig. 2). After removal of the 
whole mullerian structure, left ovary could be seen which appeared normal. On gross examination, tumor was approximately $12 \times 9 \times 8 \mathrm{~cm}$. Cut section showed whorledappearance, suggestive of leiomyoma. Histopathology confirmed the diagnosis of fibroid with hyaline degeneration (Fig. 3). Her postoperative period was uneventful. She was well at 3 months follow-up.

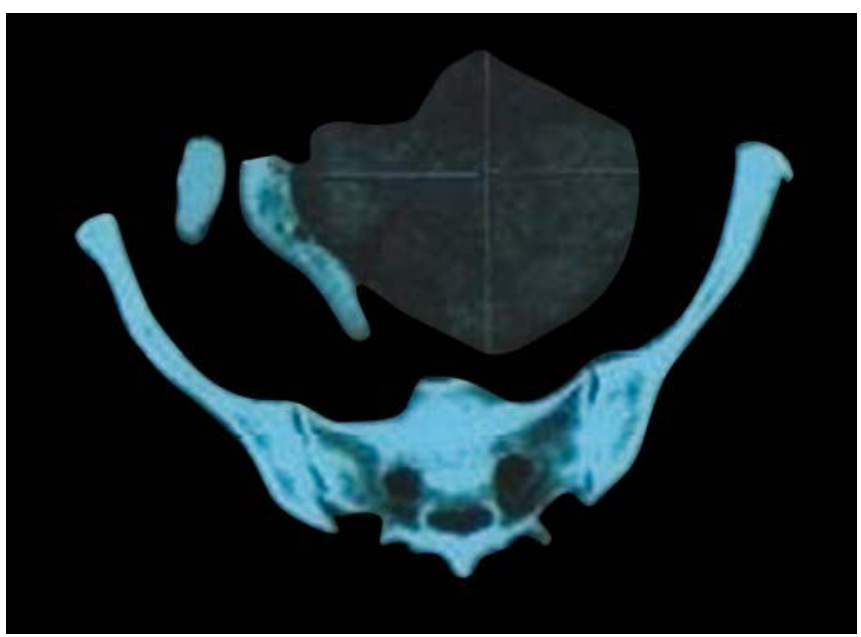

Fig. 1: Contrast enhanced CT scan picture of the pelvic tumor

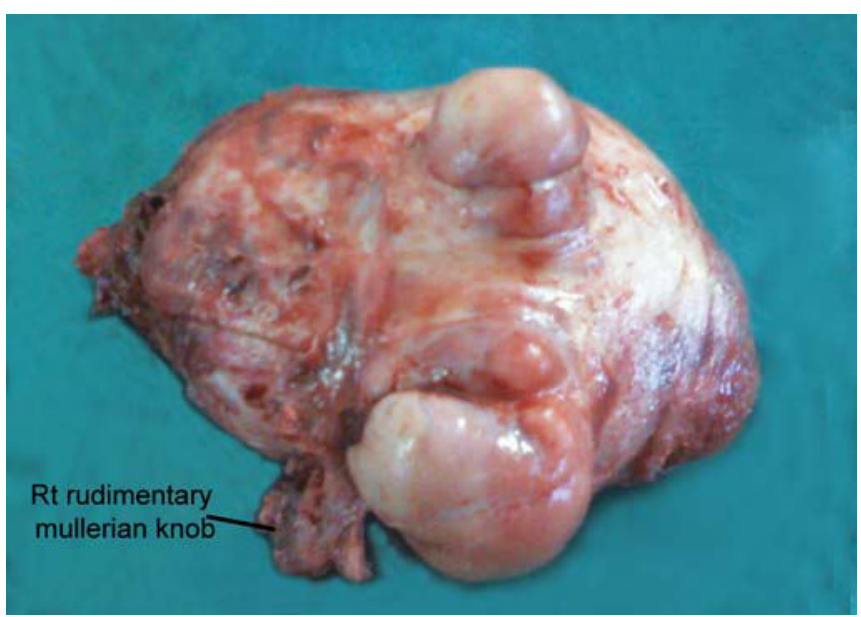

Fig. 2: Multiple leiomyomas arising from left mullerian knob alongwith the rudimentary right knob

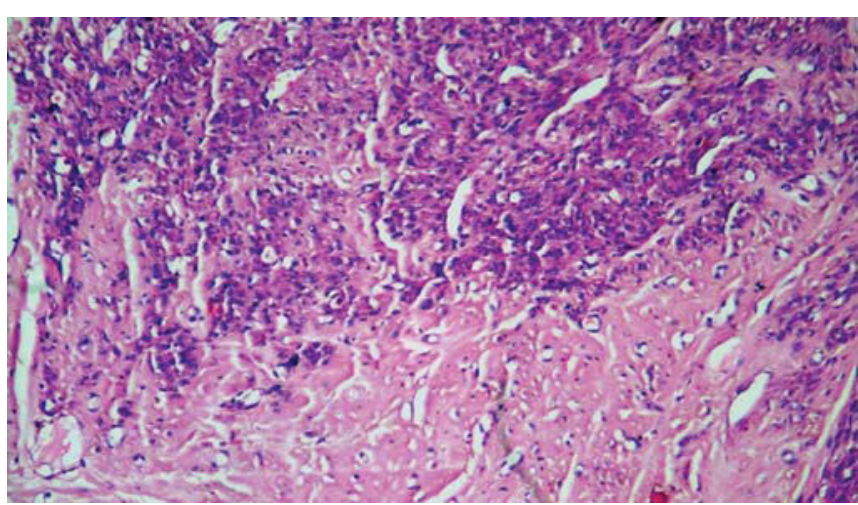

Fig. 3: Histophotomicrograph showing leiomyoma with hyaline degeneration

\section{DISCUSSION}

The MRKH syndrome is characterized by failure of fusion of the two mullerian ducts in the seventh week of embryological development which results in congenital absence of vagina and either the absence of uterine tissue or presence of two laterally situated, solid, muscular rudimentary uterine anlage/knob connected by midline, fibrous bands. Occasionally, a small palpable cord (3rd knob) may lie in the midline, but it is again nonfunctional. ${ }^{12,14}$ Rarely, endometrium can exist which becomes active under the influence of estrogen. Reports have described patients with functioning endometrial tissue or even a hematometra in one or both of the rudimentary uterine anlage. ${ }^{17}$ As ovarian function is normal, estrogen dependent pathological conditions can develop, including myomas, neoplasms and endometriosis. Theoretically fibroids can undergo degenerative changes as seen in normal uteri but only one such case has been reported in literature (fibroid with degenerative changes in a MRKH case). ${ }^{7}$

When a pelvic mass is found in MRKH cases, a laparotomy/laparoscopy is indicated and appropriate management depends on underlying pathology. The removal of the symptomatic tumor with the adjacent uterine remnant is indicated, ${ }^{5}$ which can be done laparoscopically. ${ }^{10-12}$ Surgical considerations should be done by identification of the blood supply, separation of the mass from the broad ligament and care in identifying and keeping the entire ureter. The removal of opposite uterine remnant can be performed at the same time, with the patient's preoperative consent. In our case, removal of opposite side of uterine remnant along with leiomyomas was done to prevent the risk of recurrence.

Although the development of leiomyomas from uterine remnant is a rare finding but it is still possible in patients with MRKH and it should be considered in the list of differential diagnosis of pelvic masses with mullerian dysgenesis.

\section{REFERENCES}

1. Varner RE, Younger JB, Blackwell RE. Mullerian dysgenesis. J Reprod Med 1985;30:443-50.

2. Beecham CT, Skiendzielewski J. Myoma in association with Mayer-Rokitansky-Kuster syndrome. Am J Obstet Gynecol 1977;129:346.

3. Purwar MB, Deshpande AS, Jawade SB. Rokitansky-KusterHauser syndrome with fibromyoma arising in rudimentary buds. Asia Oceana J Obstet Gynecol 1983;9:55.

4. Metzger DA, Massad LS, Piscitelli JT. Leiomyoma in a mullerian remnant: A case report. J Reprod Med Feb 1988;33(2): 246-48. 
5. Powell B, Cunnane MF, Dunn LK, Corson SL. Leiomyoma uteri in a rudimentary uterine horn in a woman with the RokitanskyKuster-Hauser syndrome: A case report. J Reprod Med 1988; 33:493.

6. Baker J, Buttini M. Fibroid arising in a mullerian duct remnant and presenting as a pelvic mass in a patient with vaginal agenesis: An unusual finding. Aust NZ J Obstet Gynecol Aug 1995;35(3): 340-41.

7. Hsu SC, Tsai EM, Wu CH, Lee JN. A mullerian duct remnant myoma misdiagnosed as ovarian cancer in a woman with vaginal agenesis — a case report. Kaohsiung J Med Sci Feb 1999;15(2): 110-02.

8. Mc Intyre G. Mullerian duct remnant fibroids: A case report. West Indian Med J June 2001;50(2):169-70.

9. Choi Manyan, Ka Ming Mok. Uterine fibroids and adenomyosis in a woman with RKH. Journal of Obstetrics and Gynecology 2002;22:561-62.

10. Tsin DA, Waters TK, Granato RC. Laparoscopic myomectomy in a patient with Mayer- Rokitansky-Kuster-Hauser syndrome. J Am Assoc Gynecol Laparosc 2000;7:411-13.

11. Jadoul P, Pirard C, Squifflet J, Smets M, Donnez J. Pelvic mass in a woman with Mayer-Rokitansky-Kuster-Hauser syndrome. Fertile Steril 2004;81:203-04.

12. Kieth Edmonds D. Multiple fibroids in a postmenopausal woman with MRKH. J Pediatr Adolesc Gynecol 2003.

13. Deligeoroglou E, Kontoravdis A, Makrakis E, Christopoulos P, Kountouris A, Creatsas G. Development of leiomyomas on the uterine remnants of two women with Mayer-Rokitansky-KusterHauser syndrome. Fertil Steril May 2004;81(5):1385-87.

14. Roy Kallol K, Lal Suman, Banerjee Neelam. Large leiomyomas in Mayer-Rokitansky-Kuster-Hauser syndrome. J Obstet Gynecol India March/April: 55;2.
15. Mittal Elly, Sengupta Rinku, Bhargava Vijay Lakshmi. Fibroid arising in a mullerian duct remnant presenting as a pelvic mass. Journal of Gynaecologic Surgery. June 2007;23(2): 79-82.

16. Lamarca Marta, Novarro Ricado, Ballestros Maria Eugenia, Garcia-Aguirre Salvador, et al. Leiomyomas in both uterine remnants in a woman with the Mayer-Rokitansky-Kuster-Hauser syndrome. Fertility and sterility Mar 2009;91(3):931.e13-15.

17. Rock JA, Baramki TA, Parmley TH, et al. A unilateral functioning uterine anlage with mullerian duct agenesis. Int J Gynecol Obstet 1980;18:99-101.

\section{ABOUT THE AUTHORS}

\section{Soma Singh (Corresponding Author)}

Fellow, Department of Reproductive Medicine, Institute of Reproductive Medicine, Kolkata, West Bengal, India, e-mail: somasingh1@yahoo.com

\section{Baidyanath Chakravarty}

Director, Department of Reproductive Medicine, Institute of Reproductive Medicine, Kolkata, West Bengal, India

\section{Manju Chakravarty}

Consultant, Department of Reproductive Medicine, Institute of Reproductive Medicine, Kolkata, West Bengal, India

\section{Astha Chakravarty}

Fellow, Department of Reproductive Medicine, Institute of Reproductive Medicine, Kolkata, West Bengal, India 